We appreciate the response to this publication feature - and welcome all contributions. Contributions may be sent to Phil Oshel, our Technical Edjtor at:

$\begin{array}{ll}\text { Mr. Phil Oshel } & \text { (608)833-2885 } \\ \text { PO Box 620068 } & \text { eMail: oshel@terracom.net } \\ \text { Middleton WI } 53562 & \end{array}$

\section{Keeping Variable Pressure SEMs Clean by Purging}

Variable pressure SEMs have become very popular in the last six years because of their ability to examine samples in the 0.1 to 2 Torr vacuum region. The advantages of this for otherwise difficult sample examinations are well known. Less known is the ability of variable pressure SEMs to clean themselves when left at low vacuum.

The basis for this cleaning effect lies in the differences in meanfree-paths of molecules at high vacuum versus those at low vacuum. At high vacuum, $<10^{-5}$ Tor, the mean free paths are greater than the dimensions of the typical SEM chamber, and the chamber is in "molecular flow" conditions where the individual free molecules move independently of each other. Pumping and removal of molecules is dependent on the ability of the pump to capture these molecules as they fly around the chamber. At low vacuum, the mean-free-path is short and the molecules move together in viscous flow. At low vacuum under active pumping, the free-contaminant molecules are caught in the flowing gas stream and are carried to the pump at high efficiency. At high vacuum free-contaminant molecules are most likely to hit a chamber surface rather than find the pump entrance where they can be captured. The efficiency of removal is simply the ratio of pump entrance area to total surface area in the chamber, which is less than $1 \%$ for common SEMs. Therefore purging a vacuum chamber with a clean gas at about 1 Tort will keep a SEM chamber cleaner than leaving it at high vacuum. (Note: There are additional considerations for ultra high vacuum systems, and this is not true for them.)

Variable pressure microscopes have purging ability for the chambers built into them. These microscopes also do not have high efficiency high vacuum pumping systems. They are built for low vacuum operation. It is common for a variable pressure SEM that is operated constantly at high vacuum to get dirty with oil backstreaming (a molecular flow phenomenon). The cure is simple: When not in use, leave the SEM in low vacuum mode and let the chamber purge itself. Backstreaming stops and the chamber cleans itself.

\section{Ronald Vane, XEI Scientific}

\section{A Sticky TEM Grid}

Very often, we wish we could have a sticky copper grid to hold our TEM samples. Here is a small trick to create sticky grids.

1) Mix a drop of 5 minutes epoxy in a petri dish

2) Dilute this epoxy in Acetone in about a 20:1 ratio

3) Immerse the copper grid you want to use into this solution

4) Remove the grid and blow air on the grid from the side to remove excess epoxy and to open up the grid openings.

That's it. Now you have a TEM grid covered with a very thin layer of 5 minutes epoxy only on grid bars.

I use this sticky grid for semiconductor TEM sample preparation to hold the epitaxial layer when the substrate is chemically removed. A sticky grid is laid down on the area of interest. Wait 5 minutes for epoxy to cure. Then I can start my selective etching process from the backside of the sample.

After etching, the thin and fragile layer of semiconductor (less than 1 $\mu \mathrm{m})$ will be held by the grid to give enough mechanical stength for handling. You can certainly think of other applications.

Hei-Ruey Harry Jen, AMP Inc.

\section{TEM of Cultured Cells on Coverslips}

For TEM of cultured cells, we grow the cultures on "Thermanox" tissue culture coverslips (from Nalge Nunc International, 50 sterile coverslips, $13 \mathrm{~mm}$ diameter is catalog 174950). The coverslips can be treated with all the same chemistry as tissue, including propylene oxide and Spurr's epoxy, which are two components which solubilize polystyrene.

Coverslips are easily processed in disposable polypropylene $50 \mathrm{~mL}$ centrifuge tubes, useful since the cell surface will not contact the wall of these conical tubes.

1) Sink the Thermanox coverslips cell side up in freshly made Spurt's.

2) Following polymerization, remove coverslips by first sawing a small area of the epoxy/ceil/substrate, then immersing in liquid nitrogen for a few seconds and prying away the substrate. The embedded cells are now on the surface of the epoxy.

3) Re-embed two fragments of the culture face to face for crosssections, or cut the block parallel to the face for tangential sections.

We particularly like the round $13 \mathrm{~mm}$ Thermanox coverslips for immunocytochemistry of cultured cells since they can be floated cell side down in a drop of $100 \mu \mathrm{L}$ antibody - gold conjugate, which conserves reagents.

If a larger culture is desired, "Permanox" culture dishes could also be used, which are equally resistant to chemicals common in TEM processing. These are also available through most EM and other suppliers.

Douglas R. Keene, Shriners Hospital Microscopy Unit, Portland, OR

\section{Cornflaking Cells}

In cytologic preparations for diagnostic cytology, cornflaking (aka the "brown artifact") is a common phenomenon, especially in Pap smears that contain many squamous epithelial cells. Caused by air trapped on the surface of superficial cells almost exclusively, these cells have a brownish crinkly appearance on their surface. It can range from a small area per cell to nearly the entire cell, and from a few cells per preparation (which is common) to nearly every cell. In the worst case, the preparation is useless and must be processed to remove the air.

Air bubbles form when xylene evaporates, leaving behind the exposed surface of these cells, which are coursed by fine grooves to promote cell attachment. Anything that promotes xylene evaporation promotes cornflaking, such as prolonged draining between rinses, coverslipping multiple slides at a time, and coverslipping under a fume hood in which air enters the hood opening at 75 to 100 linear feet per minute.

To prevent cornflaking, coverslip one slide at a time. Do not drain the slide thoroughly. Try putting a chemical splash shield at the front edge of the fume hood and coverslip behind it. The shield diverts the incoming air and creates a quite zone where evaporation occurs more slowly.

While some recommend $50 \%$ glycerin to remove the air, plain tap water works well. Since water removes the brown artifact, it is clear that this artifact does not occur before staining. Indeed, even if it did, it would be removed by the water rinses in the front half of the Pap stain and it would never be seen. Since tap water destains the orange $G(O G)$ and $E A$ dyes but not hematoxylin, restaining is required only for $O G$ and $E A$.

It is not necessary to destain the slide, as the artifact is air and not stain. Remove the cover glass, pass the slide back from xylene to absolute alcohol, then to water. Microscopically check the slide to verify the removal of the artifact, then restain beginning in OG. A preceding rinse in alcohol is unnecessary.

Gary Gill, Diagnostic Cytology Laboratories, Inc. 